\title{
TEXTURE AND ANISOTROPY OF AN Al-Li ALLOY
}

\author{
F. BARLAT, D.J. LEGE, J.C. BREM and C.J. WARREN \\ Alcoa Laboratories, Alcoa Center, PA 15069, USA
}

\section{INTRODUCTION}

The prediction of mechanical and forming properties of materials is a useful tool for material and product design. The choice of constitutive relations is one of the most critical steps in the modeling process. When these relations are based on microstructural information, they lead to accurate predictions of processes and material properties. However, since the microstructure of materials is very complex, suitable phenomenological descriptions are more convenient if they lead to reasonable results. The prediction of material behavior relies on many assumptions, and it is very important to compare and validate the applicability of these assumptions with experiments. In this sense, Al-Li alloys are especially interesting to study, because, unlike the majority of aluminum alloys, they exhibit a strong crystallographic texture. Moreover, depending on the material temper, precipitates which influence their plastic behavior may be present in the microstructure. As a consequence, the mechanical and forming properties of Al-Li alloys are strongly anisotropic. Therefore, they are ideal alloys to test anisotropic constitutive relations. In addition, there is technological interest in Al- $\mathrm{Li}$ alloys, particularly for the aerospace industry, because of their low density and high elastic modulus.

\section{PROCEDURE}

A 2090-T3E28 Al-Li sheet $(1.6 \mathrm{~mm})$ sample was characterized. The crystallographic texture was assessed with the crystallite orientation distribution function (CODF), calculated from (200) and (111) pole figures. Uniaxial tension tests were used to characterize the directionality of Young's modulus, yield stress, $R$ value. Young's modulus was defined as the slope of the uniaxial stress/strain curves. Yield stress was determined by the conventional $0.2 \%$ offset plastic strain method. The $R$ value was calculated as the ratio of the width to the thickness strain of a tensile specimen deformed at a strain of about $10 \%$. Uniaxial compression tests were performed to measure the compressive yield strength anisotropy as well as Young's modulus. Hydraulic bulge tests were conducted to get an estimate of the balanced biaxial yield stress.

The polycrystalline yield surface of the Al-Li alloy sheet was predicted from crystallographic texture data alone with the Taylor ${ }^{1} /$ Bishop and Hill $^{2}$ model. In order to describe plastic anisotropy completely (texture plus precipitates effects), the coefficients of phenomenological yield surfaces were calculated using the experimentally measured uniaxial tension and bulge test yield stresses. The $R$ and $\Gamma$ values were computed from the polycrystalline and phenomenological yield surfaces. The $\Gamma$ value was defined as the ratio of the shear strain to the longitudinal strain in uniaxial tension ${ }^{3}$. Predicted and 
experimental results were compared to appraise the validity and the limitations of each constitutive model. The microstructural features other than texture that possibly affect the anisotropic response of Al-Li sheets are discussed.

\section{PHENOMENOLOGICAL MODELS}

In classical flow theory of plasticity, the yield surface is assumed to be a potential. Hershey ${ }^{4}$ and Hosford 5 proposed a yield function based on the three principal stresses to describe the behavior of isotropic polycrystals:

$$
\phi=\left|\sigma_{1}-\sigma_{2}\right|^{m}+\left|\sigma_{2}-\sigma_{3}\right|^{m}+\left|\sigma_{3}-\sigma_{1}\right|^{m}=2 \sigma^{m}
$$

where $\sigma$ is the uniaxial flow stress. Hershey proposed the function as a good approximation of a self-consistent estimate of the yield surface for isotropic FCC polycrystals. Hosford showed that Equ. 1 reduces to the Tresca yield condition for $m=1$ or $m=\infty$ and to the Von Mises criterion for $m=2$ or $m=4$. Moreover, he showed that the behavior of BCC and FCC isotropic materials was particularly well represented for $m=6$ or $m=8$, respectively. Recently, Barlat and Lian 6 generalized this yield function for the case of orthotropic materials subjected to plane stress condition. Their tricomponent yield function is expressed as follows:

$$
\begin{aligned}
& K_{1}=\frac{\sigma_{x x}+h \sigma_{y y}}{2} \quad K_{2}=\sqrt{\left(\frac{\sigma_{x x}-h \sigma_{y y}}{2}\right)^{2}+p^{2} \sigma_{x y}^{2}} \\
& \phi=d\left|K_{1}-K_{2}\right|^{m}+a\left|K_{1}+K_{2}\right|^{m}+(2-a)\left|2 K_{2}\right|^{m}=2 \sigma^{m}
\end{aligned}
$$

$\sigma_{\mathrm{xx}}$ and $\sigma_{\mathrm{yy}}$ are the stresses in the rolling and transverse directions, respectively. $\sigma_{\mathrm{xy}}$ is the shear stress in the plane of the sheet and $a, h, p$ and $m$ are material coefficients. Even though this yield function can be used in many sheet forming problems, for which the plane stress assumption is valid, it is not a suitable description for the behavior of bulk material. So, Barlat, Lege and Brem 7 generalized the Hershey and Hosford criterion to the case of a six-component function for orthotropic materials with no restrictions on the loading conditions:

$$
\begin{aligned}
& A=\sigma_{y y}-\sigma_{z z} ; B=\sigma_{z z}-\sigma_{x x} ; C=\sigma_{x x}-\sigma_{y y} ; F=\sigma_{y z} ; G=\sigma_{z x} ; H=\sigma_{x y} \\
& I_{2}=\frac{(f F)^{2}+(g G)^{2}+(h H)^{2}}{3}+\frac{(a A-c C)^{2}+(c C-b B)^{2}+(b B-a A)^{2}}{54} \\
& I_{3}=\frac{(C C-b B)(a A-c C)(b B-a A)}{54}+f g h F G H-\frac{(c C-b B)(f F)^{2}+(a A-c C)(g G)^{2}+(b B-a A)(h H)^{2}}{6} \\
& \theta=\cos ^{-1}\left(\frac{I_{3}}{I_{2}^{3 / 2}}\right) \quad 0 \leq \theta \leq \pi \\
& \phi=\left(3 I_{2}\right)^{m / 2}\left\{\left[2 \cos \left(\frac{2 \theta+\pi}{6}\right)\right]^{m}+\left[2 \cos \left(\frac{2 \theta-3 \pi}{6}\right)\right]^{m}+\left[-2 \cos \left(\frac{2 \theta+5 \pi}{6}\right)\right]^{m}\right\}=2 \sigma^{m}
\end{aligned}
$$


In Equations (3), A, B, C, F, G and $\mathrm{H}$ are simple functions of stress components defined by the Bishop and Hill notation, and $a, b, c, f, g, h$ and $m$ are materials coefficients. When the material is isotropic, $a=b=c=f=g=h=1$ and $-3 I_{2}$ and $2 I_{3}$ reduce to the second and the third stress tensor invariant, respectively. Barlat et al. ${ }^{7}$ have shown that this yield function provides a very good description of the behavior of a 2008-T4 autobody sheet sample. The advantage of this criterion is that its formulation is relatively simple, compared to polycrystal model formulations. Therefore, it is very suitable for FEM analysis of metal forming problems.

\section{RESULTS AND DISCUSSION}

The CODF intensities of the major texture components frequently found in aluminum alloys are shown in Fig. 1 for the 2090-T3E28 sheet. The Cube and Goss annealing texture components, and the rolling components located on the $\beta$-fiber are included in this figure. The CODF of 5182-H19, a typical heavily cold rolled Al-Mg alloy, is also represented on Fig. 1 for comparison. The 2090-T3E28 sheet exhibits very strong rolling texture intensities particularly near the Brass orientation where the CODF peak value is approximately four times larger than that of 5182-H19. This illustrates the severity of the crystallographic texture usually found in Al-Li alloys.

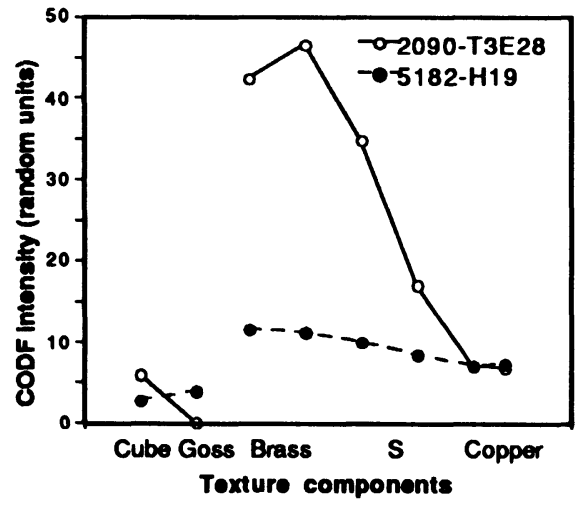

Figure 1. CODF intensity along squeleton line for 2090-T3E28 and 5182H19.

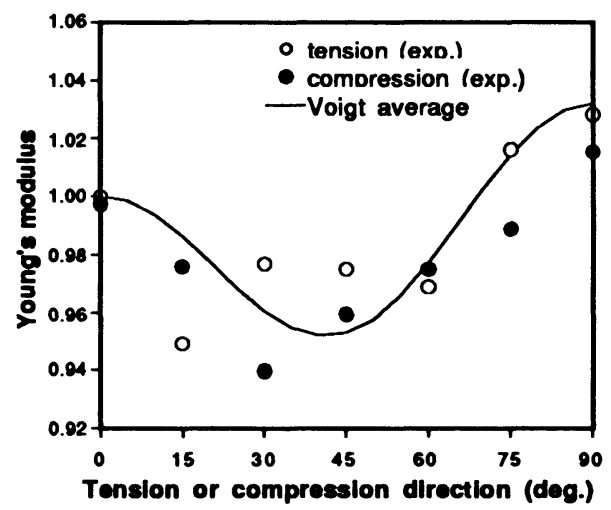

Figure 2. Young's modulus measured for uniaxial tension and compression and Voigt average for 2090-T3E28.

The Young's modulus for 2090-T3E28 was measured from the stress/strain diagrams generated during standard uniaxial tension or compression tests. While this type of measurement is not very accurate, it is a good first approximation. The same general trend appears in both tension and compression modulus data (Fig. 2). The modulus tends to decrease when the angle between rolling and loading direction increases from $0^{\circ}$ to $30^{\circ}$. As this angle increases further, the modulus increases until it is higher in the transverse than it is in the rolling direction. The Young's modulus was predicted using the CODF information, along with the values of the single crystal moduli $C_{11}, C_{44}$ and $C_{12}$ found in the literature for pure aluminum ${ }^{8}$, and for a binary $\mathrm{Al}-\mathrm{Li}$ alloy with $5 \mathrm{at} \% \mathrm{Li}$ content ${ }^{9}$. The Voigt average was calculated using the fourth order $W$ coefficients of the harmonic series representing the crystallographic texture ${ }^{10}$. The relative Young's modulus variation predicted with this model was the same for both set of $\mathrm{C}_{\mathrm{ij}}$, and it was in agreement with experiments. 


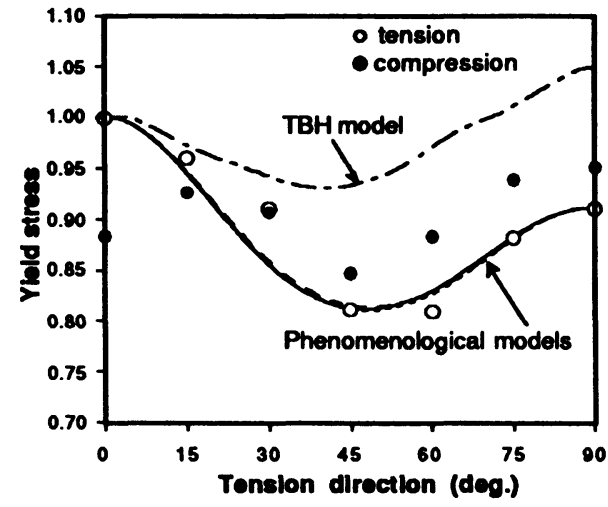

Figure 3. Predicted and experimental normalized yield stress directionality for 2090-T3E28.

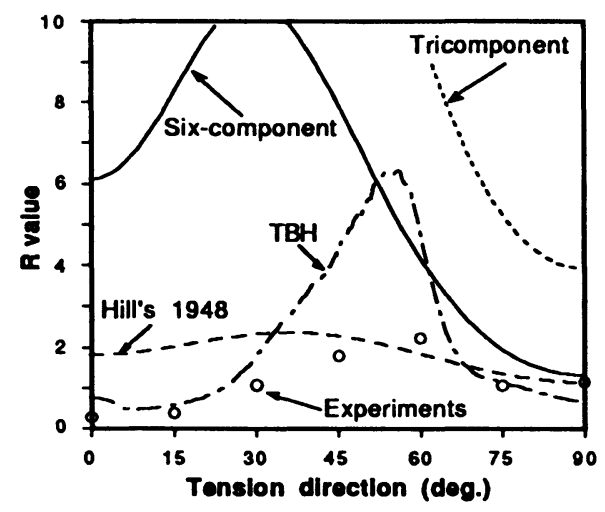

Figure 5. Predicted and experimental $R$ value directionality for 2090-T3E28.

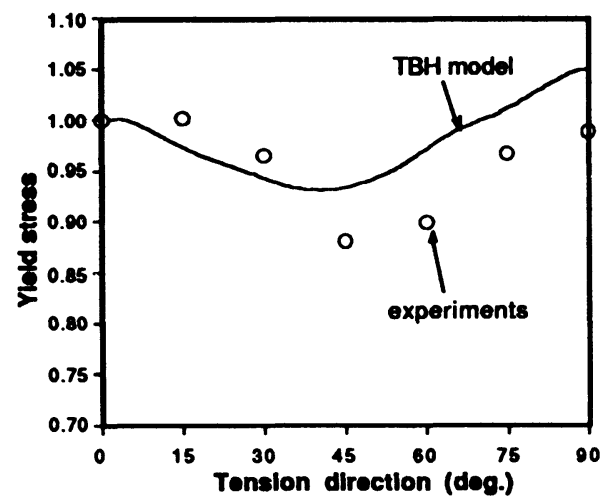

Figure 4. TBH and experimental normalized yield stress average (tensioncompression) for 2090-T3E28.

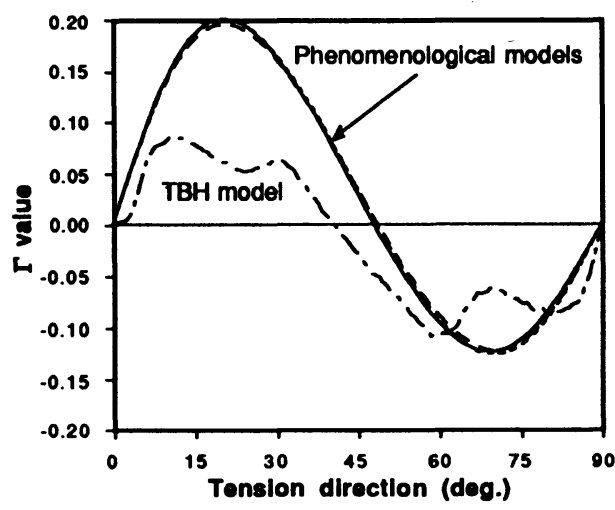

Figure 6. Predicted $\Gamma$ value directionality for 2090-T3E28.

The CODF was also used to compute the yield surface using the Taylor / Bishop and Hill model (TBH), and plastic properties in uniaxial tension were calculated using this TBH yield surface. Fig. 3 shows that the yield stress variation predicted by the TBH model exhibits a minimum near $45^{\circ}$. While this trend is in agreement with the experiments, the magnitude of the yield stress anisotropy is not very well predicted by the TBH model. The experimental yield stress in the rolling direction is used to scale the TBH predictions. If the data from the tension and compression tests are averaged as in Figure 4, the anisotropy prediction is in better agreement with experiments.

Three different phenomenological yield conditions have also been used to characterize the behavior of the 2090-T3E28 sheet sample: Hill's $1948^{11}$, the tricomponent and the six-component yield functions presented in the previous section. The constants in these criteria have been calculated to get a good approximation of the uniaxial tension yield stress directionality (Fig. 3) and of the balanced biaxial yield stress measured with the bulge test. Fig. 5 shows experimental data and predictions of the $R$ value directionality. None of the predictions is in good agreement with the experiments. Perhaps the TBH 
model gives the best predictions near the rolling and transverse directions while Hill's 1948 criterion leads to the best average $R$ value prediction.

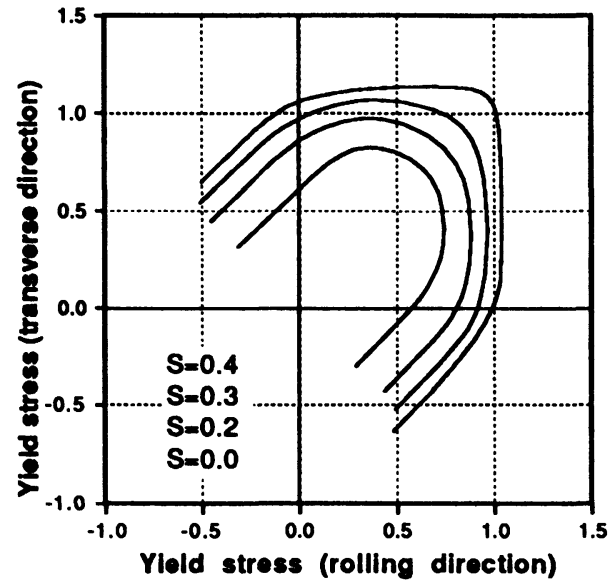

Figure 7. Taylor/Bishop and Hill yield surface for 2090-T3E28.

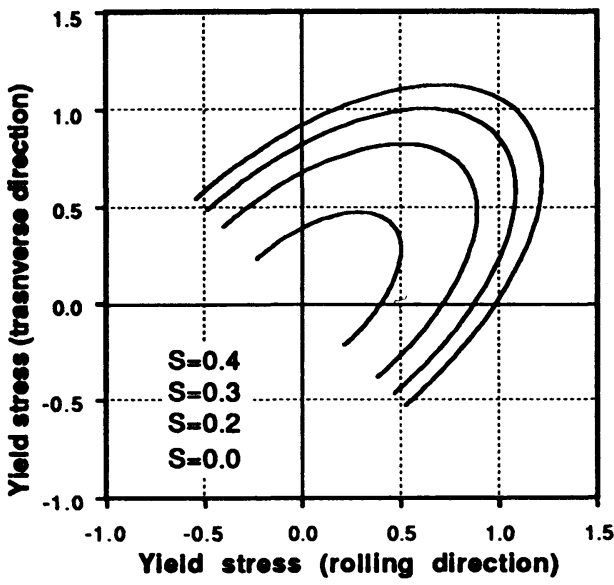

Figure 8. Hill's 1948 yield surface for 2090-T3E28.

Fig. 6 shows the prediction of directionality for the $\Gamma$ parameter. Based on the associated flow rule, BARLAT and RICHMOND ${ }^{3}$ have shown a general relationship between this parameter and the yield stress, regardless of the yield surface description. Therefore, the similarity of the predicted $\Gamma$ profile for all the phenomenological models was expected because of the similarity of the yield stress directionality (Fig. 3).

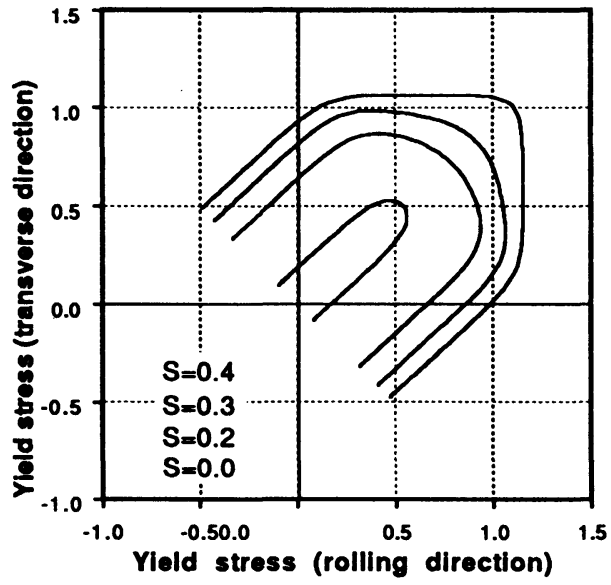

Figure 9. Tricomponent yield surface for 2090-T3E28.

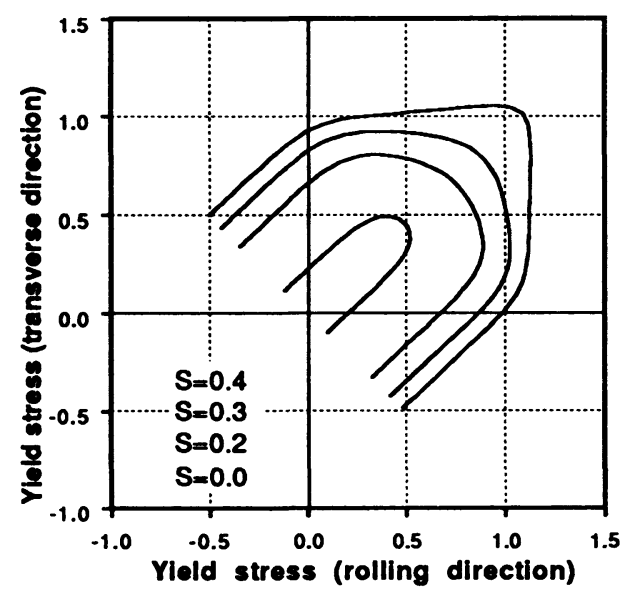

Figure 10. Six-component yield surface for 2090-T3E28.

Figure 7 shows the intersection of the TBH yield surface with planes of constant shear stress $S=\sigma_{x y}$. In this plot, all of the stresses are normalized by the uniaxial yield stress in the rolling direction. This figure shows that the TBH yield surface exhibits a 
small radius of curvature near the uniaxial and biaxial tension ranges. However, the yield surface predicted from the quadratic Hill's 1948 function does not show these small radii of curvature (Fig. 8). Figures 9 and 10 show the yield surface calculated with the tricomponent and the six-component models, respectively. The exponent in these functions is relatively high, $m=14$, which leads to a small radius of curvature in the uniaxial and biaxial stress ranges, as suggested by the TBH model.

The overall study of the results indicates that the strong texture of the 2090-T3E28 sheet leads to anisotropic properties. The directionality of Young's modulus predicted with the Voigt model using CODF data is in relatively good agreement with the experiments, while the plastic properties predicted with the Taylor / Bishop and Hill model (TBH) are not. This suggests that other microstructural features in addition to texture influence plastic anisotropy. Bate et al. ${ }^{12}$ have shown that precipitation changes the anisotropy of two-phase aluminum alloys. Since 2090-T3E28 contains precipitates, this may affect the plastic anisotropy results. The difference between tension and compression yield stresses may be due to the existence of residual stresses in the material. Precipitates tend to create a long range elastic strain field due to the misfit strains between precipitates and matrix. Thus, the tension and compression yield stresses would be expected to differ. The fact that the TBH prediction is in better agreement with the average (tension and compression) supports this residual stress argument.

The form of the tricomponent and six-component yield functions presented in this paper was suggested by polycrystal models. Therefore, with a relatively large exponent $(m=14)$, the yield surfaces calculated with these functions are similar to the TBH ones. Experimental uniaxial and biaxial yield stresses are used to calculate the constant coefficients in these phenomenological functions, but the resulting $R$ values are not well predicted. Since the uniaxial tension and compression yield stresses are not similar, as a first approximation, the yield surface may be considered to be translated in stress space. When the yield surface curvature is small in the uniaxial range, the strain rate increment normal to the surface, and consequently the $R$ value, may change drastically for a small translation. This may explain the disagreement between theoretical and experimental $\mathbf{R}$ values. Nevertheless, these phenomenological yield functions are believed to be useful, because they take the crystalline nature of the material into account through the parameter $\mathrm{m}$, and the material coefficients are calculated from actual test results.

\section{REFERENCES}

1. G.I. Taylor, J. Inst. Metals, 62,307 (1938).

2. J.W.F. Bishop and R. Hill, Phil. Mag., 42, 414 and 1298 (1951).

3. F. Barlat.and O. Richmond, Mat. Sci. Eng., 95, 15 (1987).

4. A.V. Hershey, J. Applied Mechanics, 76, 241 (1954).

5. W.F. Hosford, J. Applied Mechanics, 39, 607 (1972).

6. F. Barlat and J. Lian, Int. J. Plasticity, 5, 51 (1989).

7. F. Barlat, D.J. Lege and J.C. Brem, accepted for publication in Int. J. Plasticity (1990).

8. F. Milstein and D. Rasky, Phys. Rev. B, 33, 2341 (1986).

9. W. Müller, E. Bubeck and V. Gerold, Proc. 3rd Aluminum-Lithium Conference, (The Institute of Metals, London 1986), p. 435.

10. G.J. Davies, D.J. Goodwill and J.S. Kallend, Metal. Trans., 3, 1627 (1972).

11. R. Hill, Proc. Soc. London, Ser. A, 193, 281 (1948).

12. P. Bate, W.T. Roberts and D.V. Wilson, Acta Metal., 29, 1797 (1981). 